\title{
The IMF and star-formation history of the ionizing cluster of 30 Doradus
}

\author{
Fernando J. Selman, Jorge Melnick, Guillermo L. Bosch, \\ and Roberto J. Terlevich \\ ESO, Alonso de Córdova 3107, Santiago, Chile
}

\begin{abstract}
A new method is used to determine masses, ages, and reddening for the stars of the starburst cluster NGC 2070 in 30 Doradus. The data are consistent with a star-formation rate which increases exponentially with a characteristic rise time of $1.7 \mathrm{Myr}$, independent of mass, starting $7 \mathrm{Myr}$ ago. The IMF is consistent with a single power law IMF with Salpeter slope over the whole $3 \mathrm{M}_{\odot}<M<120 \mathrm{M}_{\odot}$ range investigated, with evidence for flattening for the innermost radial bin.
\end{abstract}

\section{Overview}

We have applied a new method of analysis to $U B V$ data obtained with the ESONTT telescope under excellent seeing conditions (Selman et al. 1999). With this method we assign ages, reddening, and masses to a sample of stars within $90^{\prime \prime}$ of the center of the cluster. We have used a catalogue of newly obtained plus published spectral types to determine that this photometric method can assign masses in an unbiased way up to the most massive stars observed. The only drawback of the method is that the effective temperatures, masses, and ages thus determined are dependent on stellar evolutionary models. The standard method previously used to determine physical parameter introduced model dependencies only on the masses and ages, not on the effective temperatures (Melnick 1985). Because we are mainly interested in the IMF and ages we do not see this as a particularly troublesome disadvantage. In any case, for the most massive stars we have spectral types for most of them.

Two insidious systematic effects are found to affect the analysis of the data using the standard method: (1) there is migration of stars to different mass bins creating the ubiquitous hot plume of stars in the theoretical HR diagrams; and (2) the differential reddening introduces a systematic effect for the stars that are close to the limit of the photometry. The present method has allowed us to get rid of these effects using Bayesian analysis of the $V,(B-V)$, and $(U-B)$ data simultaneously. This has permitted us to present in Figure 1a the IMF for almost the whole intermediate to high mass range.

\section{Summary}

The most important results are:

(a) In the mass interval $3 \mathrm{M}_{\odot}<M<120 \mathrm{M}_{\odot}$ the IMF is well represented by a single power law with Salpeter exponent $(\Gamma=-1.35)$. 

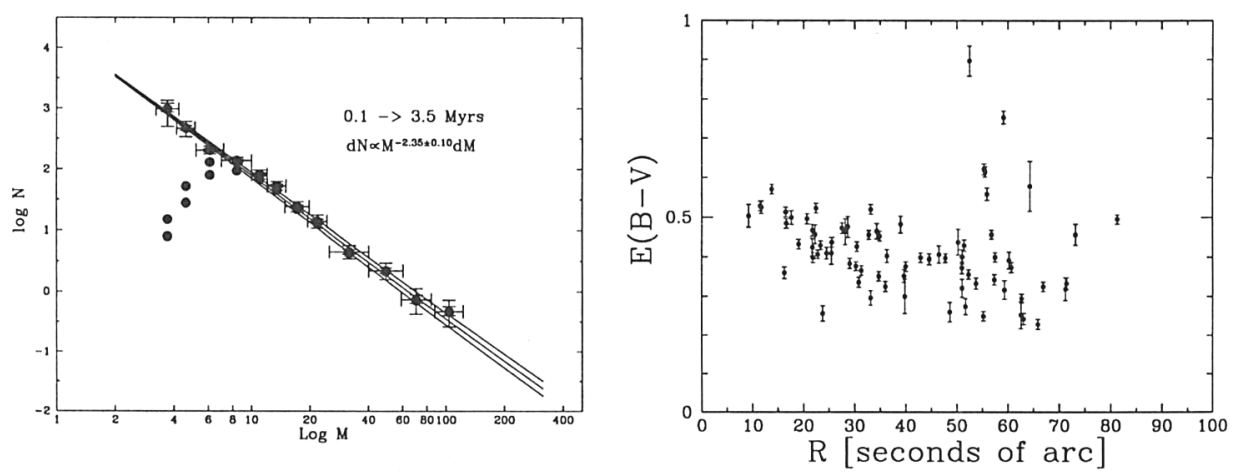

Figure 1. Left: Initial Mass Function for the whole sample of 30 Doradus stars (no radial binning). The three sets of points represent the raw counts, the counts corrected for incompleteness only, and the counts corrected for the reddening induced magnitude limit effect. Right: Reddening vs. radial distance for stars with spectroscopy.

(b) Our data show marginal evidence of flattening in our innermost radial bin $(\Gamma=-1.05 \pm 0.25)$. This radial bin is equivalent to the outermost bin of the HST intermediate mass-range work by Hunter et al. (1996), who present a best fit slope almost identical to ours, $\Gamma=-1.01 \pm 0.07$. It should be pointed out here that the Hunter et al. (1996) work is not consistent with the large number of massive stars found by Massey \& Hunter (1998) if we assume a Salpeter slope: it falls short by a factor of 2.5. Only when we use the best-fit slope of the intermediate mass-range data, then the predicted number of high-mass stars agrees with that observed: the mass segregation appears to be real.

(c) The photometry reveals two reddening components (Figure 1b): the first one is the expected highly variable component, apparently associated with the fragmented shell of swept and/or ejected gases; and the second one is a smoother, more moderate, also variable component which increases toward the center associated and mixed with the cluster itself. The presence of this component is somewhat puzzling, because it reveals the presence of dust down to the very core of the cluster, where we would have expected depletion. Although we can only speculate as to its origin, several possibilities come to mind: (i) the sweeping process has failed to get rid of all the gas; or (2) the gas has been recently formed in colliding winds; or (3) the dust is being ablated from the remnants of gas and dust disks presumably still present around a fraction of newly formed stars.

\section{References}

Hunter, D.A., O'Neil, E.J., Lynds, R., Shaya, E.J., Groth, E.J., Holtzman, J.A. 1996, ApJ 459, L27

Massey, P., Hunter, D.A. 1998, ApJ 493, 180

Melnick, J. 1985, A\&A 153, 235

Selman, F.J., Melnick, J., Bosch, G., Terlevich, R.J. 1999, A\&A 341, 98 